Open Access

\title{
Volume-replacement ratio for crystalloids and colloids during bleeding and resuscitation: an animal experiment
}

\author{
Ildikó László ${ }^{2 *} \mathbb{D}$, Gábor Demeter ${ }^{2 \dagger}$, Nándor Öveges ${ }^{2}$, Dániel Érces ${ }^{1}$, József Kaszaki ${ }^{1}$, Krisztián Tánczos ${ }^{2}$
} and Zsolt Molnár ${ }^{2}$

\author{
* Correspondence: \\ laszlo.ildiko@med.u-szeged.hu \\ ${ }^{\dagger}$ Equal contributors \\ ${ }^{2}$ Faculty of Medicine; Department of \\ Anaesthesiology and Intensive \\ Therapy, University of Szeged, 6. \\ Semmelweis st, Szeged 6725, \\ Hungary \\ Full list of author information is \\ available at the end of the article
}

\begin{abstract}
Background: Fluid resuscitation remains a cornerstone in the management of acute bleeding. According to Starling's "Three-compartment model", four-times more crystalloids have the same volume effect as colloids. However, this volumereplacement ratio remains a controversial issue as it may be affected by the degradation of the endothelial glycocalyx layer, a situation often found in the critically ill. Our aim was to compare colloid and crystalloid based fluid resuscitation during an experimental stroke volume index (SVI) guided hemorrhage and resuscitation animal model.
\end{abstract}

Methods: Anesthetized and mechanically ventilated pigs were randomized to receive a colloid (Voluven ${ }^{\circledR}, \mathrm{HES}, \mathrm{n}=15$ ) or crystalloid (Ringerfundin ${ }^{\circledR}, \mathrm{RF}, \mathrm{n}=15$ ) infusion. Animals were bled till baseline SVI (Tbsl) dropped by 50\% (T0), followed by resuscitation until initial SVI was reached (T4) in four steps. Invasive hemodynamic measurements, blood gas analyses and laboratory tests were performed at each assessment points. Glycocalyx degradation markers (Syndecan-1/hematocrit ratio, Glypican/hematocrit ratio) were determined at Tbsl, T0 and T4.

Results: Similar amounts of blood were shed in both groups (HES group: 506 \pm 159 mls blood, RF group: 470 $\pm 127 \mathrm{mls}$ blood). Hemodynamic changes followed the same pattern without significant difference between the groups. Animals received significantly less resuscitation fluid in the HES compared to the RF-group: 425 [320665], vs 1390 [884-1585] mls, $p<0.001$. The volume replacement ratio was 0.92 [0.791.54] for HES; and 3.03 [2.00-4.23] for the RF-group ( $p<0.001)$. There was no significant difference between the groups in the glycocalyx degradation markers.

Conclusion: In this moderate bleeding-resuscitation animal model the volumereplacement ratio for crystalloids and colloids followed similar patterns as predicted by Starling's principle, and the glycocalyx remained intact. This indicates that in acute bleeding events, such as trauma or during surgery, colloids may be beneficial as hemodynamic stability may be achieved more rapidly than with crystalloids.

Keywords: Colloid, Crystalloid, Volume-replacement ratio, Glycocalyx 


\section{Background}

Acute bleeding is a perilous condition requiring immediate intervention before hypoperfusion leads to severe organ damage and multiple organ dysfunction. In addition to the surgical control of bleeding, fluid resuscitation remains one of the most important life-saving interventions. The use of colloids and crystalloids for resuscitation of bleeding patients has previously remained controversial with no definitive answer for the best course of action [1-4]. In trauma patients, hemorrhage has been proposed as the second most common contributing cause of death within $48 \mathrm{~h}$ following the injury $[5,6]$. In a multi-center analysis by Hoyt et al., hemorrhage was the primary cause of intraoperative death in $82 \%$ of patients with major trauma [7]. To avoid the lethal consequences of severe bleeding, intravenous fluid resuscitation is the first line of treatment, which has to be fast and efficient.

Fundamentally, crystalloids or colloids can be used for this purpose. However, ever since colloids appeared on the scene, debate over their efficacy and potential advantages over crystalloids has continued. According to Starling's "three-compartment model," crystalloids, with their sodium content similar to that of the serum, are distributed in the extracellular space, while colloids should remain intravascularly due to their large molecular weight. Therefore, theoretically four times more crystalloids should have the same volume expanding effect as colloids [8]. However, crystalloid overload can also have detrimental effects; therefore, using the right kind of fluid in appropriate amounts at the right time might improve patient outcome [9].

Nevertheless, several studies including thousands of critically ill patients have seemingly disapproved the Starling principle [10-15], concluding that there were only marginal differences in the administered volume of crystalloid and colloid solutions. However, these results might have been affected by the fact that most of the included patients were septic in whom the endothelial glycocalyx layer is often found to be impaired or destroyed, resulting in increased capillary permeability. Hence, colloids may disappear into the interstitial space in larger volumes than when the glycolcalyx is intact $[16,17]$. Furthermore, as reported in recent prospective studies $[18,19]$, non-survivor trauma patients also had significantly higher circulating syndecan-1 concentrations than survivors, indicating an impairment in the endothelial glycocalyx $[16,20,21]$. These results suggest that critical illness in general predisposes the patient to glycocalyx damage; hence, the volume-replacement ratio of crystalloids and colloids may be different from what would have been expected.

Therefore, the main aim of the current study was to compare the volumereplacement effects of crystalloid and colloid solutions during bleeding-resuscitation with moderate hemorrhage in an experimental animal model.

\section{Methods}

The experiments were performed on the EU Directive 2010/63/EU for the protection of animals used for experimental and other scientific purposes and carried out in strict adherence to the NIH guidelines for the use of experimental animals. The experimental project was approved by the National Scientific Ethical Committee on Animal Experimentation (National Competent Authority), Hungary, with license number: V./142/2013. The study was conducted in the research laboratory of the Institute of Surgical Research in a manner that did not inflict unnecessary pain or discomfort upon the animals. 


\section{Animals and instrumentation}

Vietnamese pot-bellied pigs $(n=30)$ underwent a 12-h preoperative fasting period with free access to water. The pigs were randomized into two groups: balanced crystalloid Ringerfundin, RF group (B. Braun AG) and a colloid (Voluven', hydroxyethyl starch (HES)) group. Anesthesia was induced by intramuscular injection of a mixture of ketamine $(20 \mathrm{mg} / \mathrm{kg})$ and xylazine $(2 \mathrm{mg} / \mathrm{kg})$, maintained by a continuous intravenous propofol infusion $(6 \mathrm{mg} / \mathrm{kg} / \mathrm{h}$ i.v.), and analgesia was performed with nalbuphine $(0.1 \mathrm{mg} / \mathrm{kg})$. Tracheal tubes were inserted in all animals, and the lungs were mechanically ventilated by Dräger Evita XL (Dräger, Lübeck, Germany). Tidal volume was adjusted to $10 \mathrm{~mL} / \mathrm{kg}$, and the respiratory rate was initialized to keep the end-tidal carbon dioxide and partial pressure of arterial carbon dioxide within physiological range (35-45 $\mathrm{mmHg})$. The adequacy of anesthesia was assessed by checking jaw stiffness. After induction of anesthesia, catheters were inserted into the right jugular vein, the left carotid artery, and the right femoral artery via aseptic dissection of the vessels. For invasive hemodynamic monitoring, a transpulmonary thermodilution catheter (PiCCO, PULSION Medical Systems SE, Munich, Germany) was placed in the right femoral artery $(3 \mathrm{~mm})$. A central venous catheter was implanted into the right jugular vein and was positioned by the guidance of intracavital ECG. Throughout bleeding, blood was drained through a catheter from the left carotid artery to a cylinder. An external warming device was used to retain the animals' body temperature at $37 \pm 1{ }^{\circ} \mathrm{C}$.

\section{Experimental protocol}

We applied a model which has been tested and reported in our previous experiments $[22,23]$. The study protocol is summarized in Fig. 1. Briefly, after instrumentation, 30 min was allowed for stabilization before baseline $\left(T_{\text {bsl }}\right)$ measurements were taken. At each assessment point, hemodynamic measurements, blood gas analyses, and laboratory tests were performed. After $T_{\mathrm{bs}}$, the pigs were bled until the stroke volume index dropped to $50 \%$ of its baseline value $\left(T_{0}\right)$; then, measurements were repeated.

The difference of stroke volume index (SVI) at $T_{\mathrm{bsl}}$ and $T_{0}$ was divided into four equal target values, which was planned to be reached in four steps during fluid resuscitation $\left(T_{1-4}\right)$ to reach the initial SVI by $T_{4}$. Fluid replacement was executed with boluses of balanced RF or HES solutions until the target SVI value was reached. After reaching each step, $20 \mathrm{~min}$ was allowed for equilibrium; then, blood gas and hemodynamic parameters were measured. All of the pigs were euthanized with sodium pentobarbital at the end of the experiment.

\section{Hemodynamic monitoring and blood gas sampling}

Cardiac function (CFI), cardiac index (CI), left ventricular contractility (dPmax), global end-diastolic volume (GEDI), heart rate (HR), mean arterial pressure (MAP), pulse pressure variation (PPV), stroke volume index (SVI), and stroke volume variation (SVV) were measured via transpulmonary thermodilution and pulse contour analysis at baseline and at the end of each step. All hemodynamic parameters were indexed for body surface area or body weight. Ten milliliters of less than $8{ }^{\circ} \mathrm{C}$ cold isotonic saline was injected through the jugular catheter for thermodilution-based measurements, and the average of three boluses recorded at the end of each interval. Central venous 


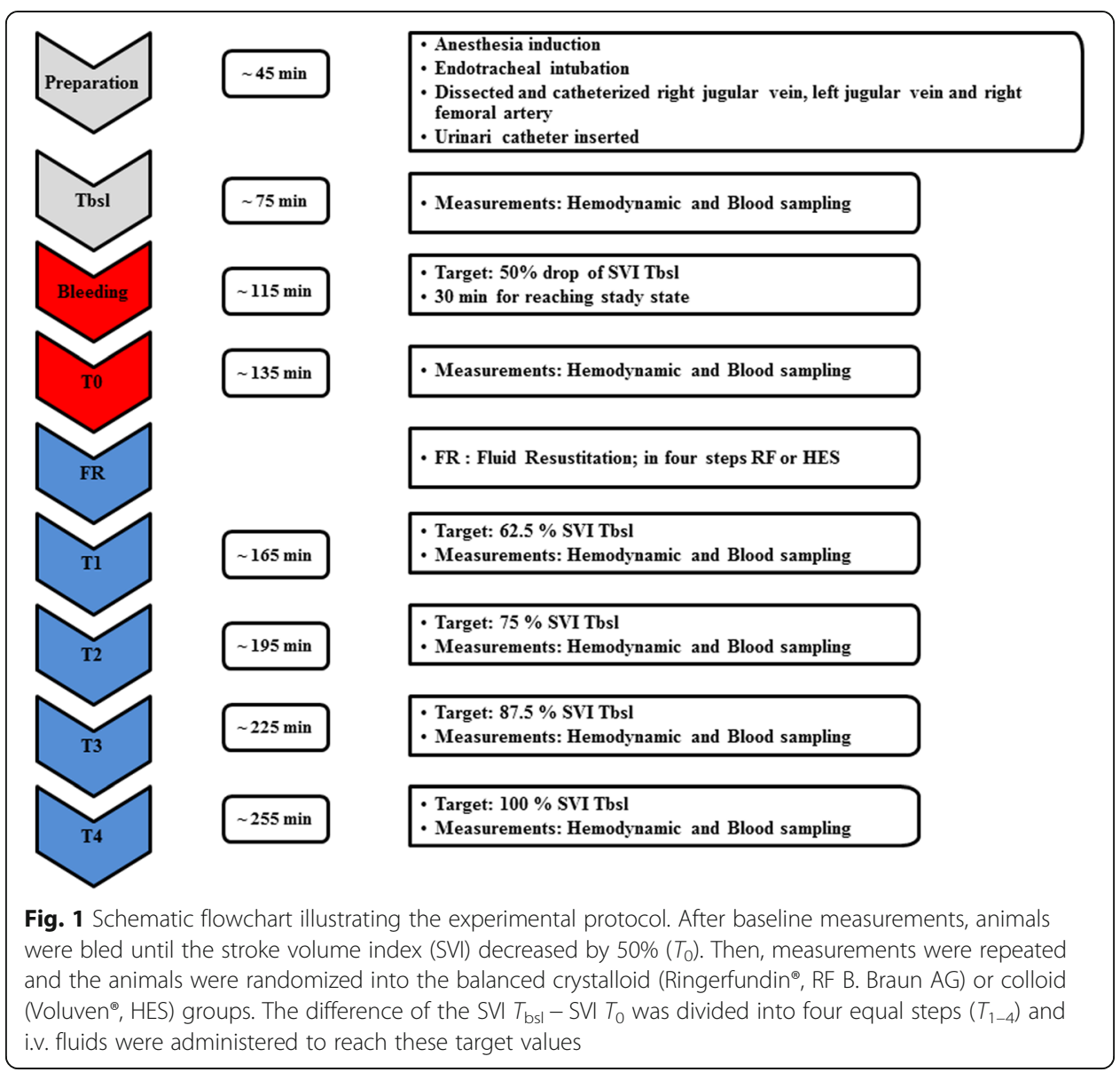

pressure (CVP) was measured via the jugular catheter in parallel with the other hemodynamic parameters. For blood gas measurements, the right femoral artery served as the site for arterial blood gas sampling and the catheter in the internal jugular vein was used for taking central venous blood gas samples. These were analyzed in parallel by co-oximetry (Cobas b 221, Roche Ltd., Basel, Switzerland) at baseline and at the end of each resuscitation step. From these parameters, the following variables were calculated [24]:

\section{Oxygen consumption $\left(\mathrm{VO}_{2}\right)$}

$$
\mathrm{VO}_{2}=\mathrm{CI} \times\left(\mathrm{CaO}_{2}-\left(\mathrm{Hb} \times 1.34 \times \mathrm{ScrO}_{2}+0.003 \times \mathrm{PcvO}_{2}\right)\right)
$$

Oxygen delivery $\left(\mathrm{DO}_{2}\right)$

$$
\mathrm{DO}_{2}=\mathrm{CI} \times(\mathrm{Hb} \times 1.34 \times \mathrm{SaO} 2+0.003 \times \mathrm{PaO} 2)
$$

$$
\text { Oxygen extraction }=\mathrm{VO}_{2} / \mathrm{DO}_{2}
$$

Volume-replacement ratios were calculated by the resuscitation fluid over the total blood loss. 


\section{Glycocalyx degradation}

Blood concentrations of syndecan-1 and glypican were quantified by enzyme-linked immunosorbent assay (ELISA) (MybioSource, Inc., San Diego, USA). For this purpose, blood samples were taken at $T_{\mathrm{bsl}}, T_{0}$, and $T_{4}$; then, the blood was centrifuged and the serum stored at $-80{ }^{\circ} \mathrm{C}$.

\section{Data analysis and statistics}

For statistical analysis, Statistical Program for Social Sciences version 23.0 for Windows (SPSS, Chicago, IL, USA) was used, and $p<0.05$ was considered significant. Data are presented as mean \pm standard deviations or median and interquartile range (IQR), respectively. For testing normal distribution, the Kolmogorov-Smirnov test was used. Independent samples were tested by independent sample $T$ test or Mann-Whitney $U$ test, as appropriate. Changes in repeated measures throughout the experiment were tested by two-way repeated measures analysis of variance (ANOVA) with Bonferroni post hoc comparisons. Categorical data were compared using $\chi^{2}$ tests. The type I error probability associated with this test of this null hypothesis is 0.05 .

\section{Results}

Out of the 30 animals, 27 survived the full experiment. Two in the HES group and one in the RF group had a sudden cardiac arrest after induction of anesthesia for reasons unknown. Therefore, the results of 27 animals (HES $n=13$; RF $n=14$ ) were finally analyzed. Demographics and overall data on fluid management are summarized in Table 1. Animals were of similar weight, height, and body surface area in both groups. For a 50\% decrease in SVI, a similar amount of blood had to be drained in both groups. Invasive hemodynamic (PiCCO) measurements were taken at similar frequencies in both groups. Urine output was significantly higher in the RF group.

\section{Macro-hemodynamic effects of fluid resuscitation}

Hemodynamic results were similar at $T_{\mathrm{bsl}}$, and goals of $50 \%$ reduction in SVI were reached by $T_{0}$ in both groups (Table 2). Hemodynamic changes during the experiment did not show clinically relevant differences between the groups. At $T_{\mathrm{bsl}}$, the SVI values

Table 1 Demographics, blood loss, and fluid therapy

\begin{tabular}{llll}
\hline & HES $(n=13)$ & RF $(n=14)$ & $p$ \\
\hline Weight $(\mathrm{kg})$ & $26.0[22.5-28.0]$ & $25.5[24.0-37.0]$ & 0.280 \\
Height $(\mathrm{cm})$ & $118.0[112.5-120.0]$ & $115.0[110.0-125.0]$ & 0.981 \\
BSA $\left(\mathrm{m}^{2}\right)$ & $0.91[0.855-0.97]$ & $0.94[0.975-1.115]$ & 0.401 \\
Shed blood $(\mathrm{mL})$ & $505.6 \pm 159.3$ & $469.7 \pm 127.3$ & 0.529 \\
Total blood loss $\left(\mathrm{mL} / \mathrm{m}^{2}\right)$ & $552.8 \pm 174.9$ & $481.1 \pm 95.2$ & 0.197 \\
PiCCO measurements $(n)$ & $23 \pm 8$ & $25 \pm 5$ & 0.422 \\
Saline used for PiCCO & $230.0 \pm 81.5$ & $252.1 \pm 58.2$ & 0.422 \\
measurements $(\mathrm{mL})$ & & & \\
Urine $(\mathrm{mL})$ & $450[350-626]^{\#}$ & $759.5[421-1110]$ & $<0.001$ \\
\hline
\end{tabular}

Data are presented as mean \pm standard deviation or median [IQR] ${ }^{\#} p<0.05$ significantly different between groups 


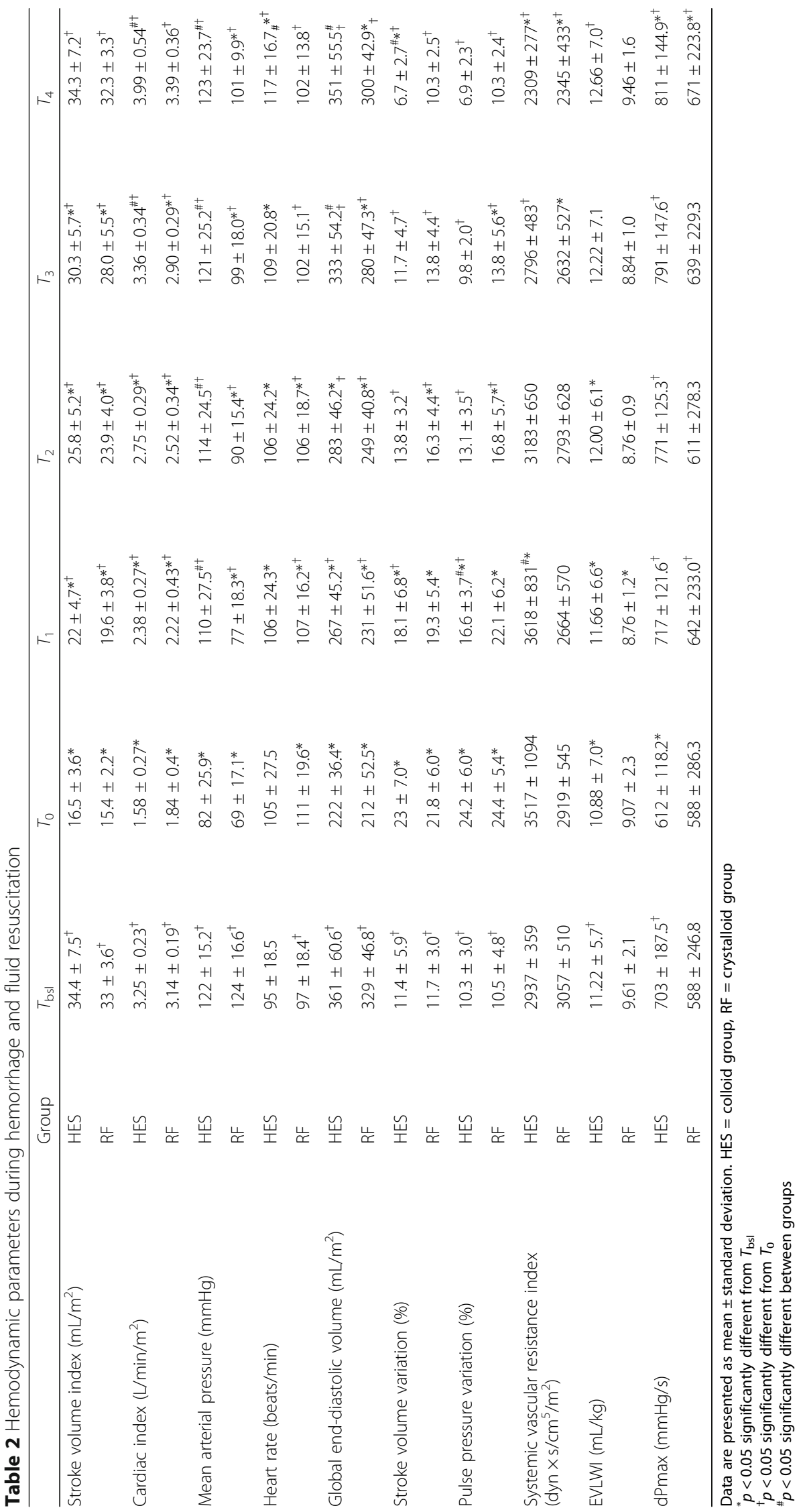


were similar, after bleeding SVI decreased by the planned $50 \%$ to $T_{0}$ and returned to its initial value by $T_{4}$.

Kinetics of the CI, MAP, HR, and GEDI showed similar pattern in both groups with significantly higher values in the HES group at the end of the experiment $\left(T_{4}\right)$. SVV and PPV almost doubled after bleeding in both groups and then returned to baseline values, being significantly lower in the HES group. Extravascular lung water index showed some changes during the experiment in both groups, without any significant differences between the groups. Contractility, as indicated by dPmax values, also showed similar changes in both groups.

\section{Changes in $\mathrm{VO}_{2} / \mathrm{DO}_{2}$ during fluid resuscitation}

Blood gas parameters during hemorrhage and fluid resuscitation are summarized in Table 3. Arterial $\mathrm{pH}$ was elevated in both groups due to unintentional hyperventilation which was then corrected towards the end of the experiment. Partial pressure of arterial oxygen tension and oxygen saturation remained stable and within the normal range throughout the study. Central venous oxygen saturation fell during the bleeding phase in both groups, but baseline values were achieved earlier in the HES group. Changes in oxygen extraction followed a similar pattern in both groups. Venous to arterial carbon dioxide gap increased significantly after the bleeding phase, with significantly higher values in the RF group, and then returned to physiological values by $T_{3}$ in both groups.

\section{Volume-replacement ratios}

While the hemodynamic profile was very similar, there were significant differences between the groups in the total amount of fluid required and in the ratio of the resuscitation fluid over the total blood loss. Significantly more RF was used during resuscitation than HES (Fig. 2). Calculating the volume-replacement ratio, it was significantly higher in the RF group, where almost three times more RF was required to achieve the same hemodynamic parameters (Fig. 3).

\section{Endothelial function}

Plasma concentration of syndecan-1 was significantly lower in the RF group at $T_{0}$ and $T_{4}$ between $T_{\text {bsl }}$ values (Fig. $4 \mathrm{a}$ ). Values of glypican in the RF group were significantly lower at $T_{4}$ compared to $T_{\mathrm{bsl}}$ and $T_{0}$ (Fig. 4b). However, the syndecan-1 hematocrit ratio and the glypican hematocrit ratio showed no significant differences throughout the whole experiment (Fig. 4c-d).

\section{Discussion}

The main findings of our study are that stable hemodynamic parameters were achieved by significantly more RF than HES boluses and that the volume-replacement ratio was more than three times higher in the RF group compared to the HES group.

The results of recently performed large controlled, randomized trials on fluid therapy in the critically ill have resulted in the development of several reviews and guidelines [25-27]. Despite the vast amount of evidence on this topic, the saga of the crystalloidcolloid controversy remains an ongoing issue. 


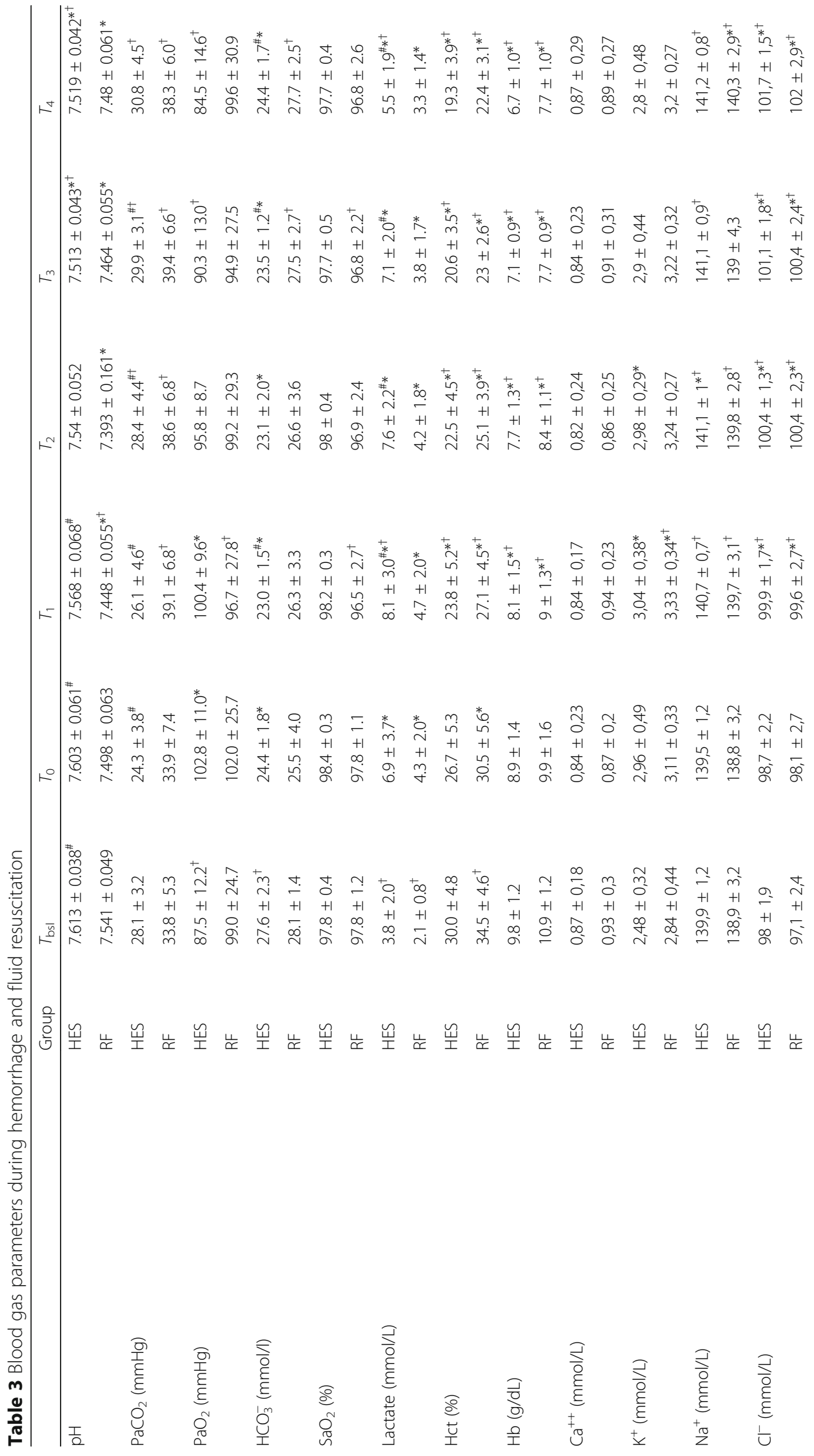




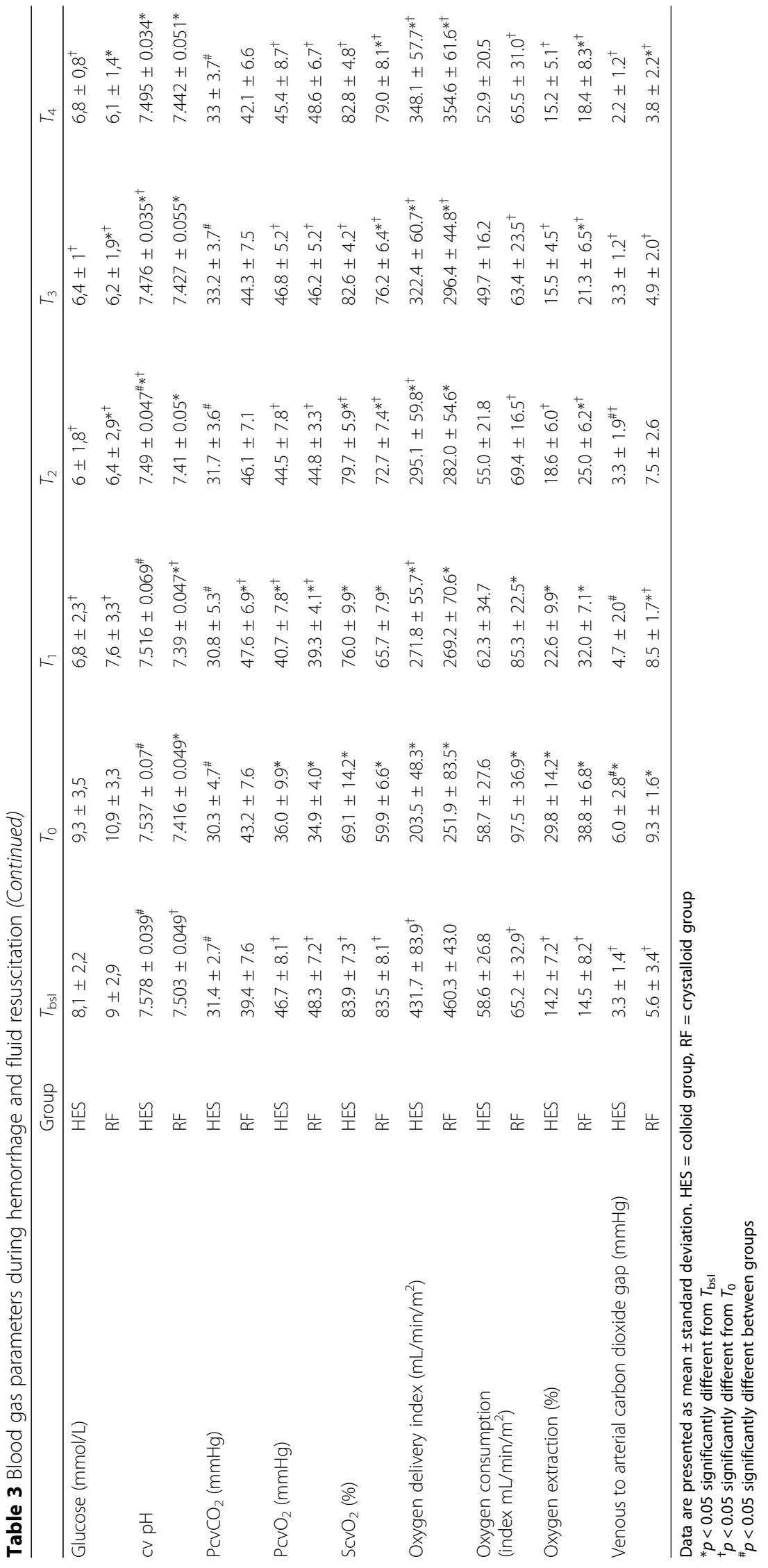




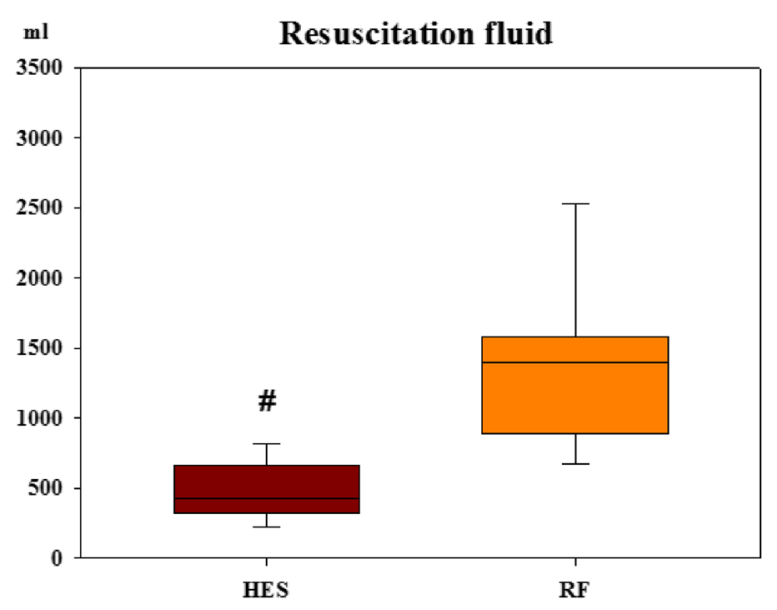

Fig. 2 Resuscitation fluid (milliliters). Data are presented as median [IQR]. $p=0.002$

One of these landmark trials was the SAFE study, where investigators compared the safety of albumin to normal saline in ICU patients $(n=6997)$. Results showed no significant difference between the groups in the hemodynamic resuscitation end points, including mean arterial pressure or heart rate, although the use of albumin was associated with a significant but clinically small increase in central venous pressure. The study showed no significant difference between albumin and normal saline regarding 28-day mortality rate or development of new organ failure [15]. SAFE was followed by the VISEP (2008), CHEST (2012) and 6S (2012) trials [11-13]. Results showed a strong association between acute kidney injury, increased use of renal replacement therapy, and the use of hydroxyethyl starch solution, which was also accompanied with unfavorable patient outcomes. The fact that a high fraction of HES solution is deposited in the tissues [10-14] might explain the impaired organ function. On the contrary, in the Colloids Versus Crystalloids for the Resuscitation of the Critically Ill (CRISTAL) trial-which was designed to test mortality related to colloid- and crystalloid-based fluid replacement in ICU patients-investigators detected a difference in death rate after 90 days, favoring the use of colloids. Furthermore, patients spent significantly fewer days on mechanical ventilation and needed shorter durations of vasopressor therapy in the colloid group compared to the crystalloid group [10].

Regarding the volume-replacement effects, in these trials, there was a similar volume-replacement ratio for crystalloids and colloids, which is summarized in Table 4. Based on these results, a common view was formed that starch solutions do not have as high potency for volume expansion as crystalloids do, but carry a greater risk of renal dysfunction and mortality. This resulted in a dramatic decrease in synthetic colloid usage around the world.

However, it is important to note that none of these trials used detailed hemodynamic monitoring. The administration of i.v. fluids was mainly based on the clinicians' subjective decision, or on parameters such as heart rate, blood pressure, central venous saturation, urine output, and lactate levels, none of which are a good predictor of fluid responsiveness. Linton et al. nicely showed in a postoperative critical care population that the relationship between MAP and oxygen delivery is very poor [28]. Therefore, one cannot exclude that a considerable number of these patients were not hypovolemic 


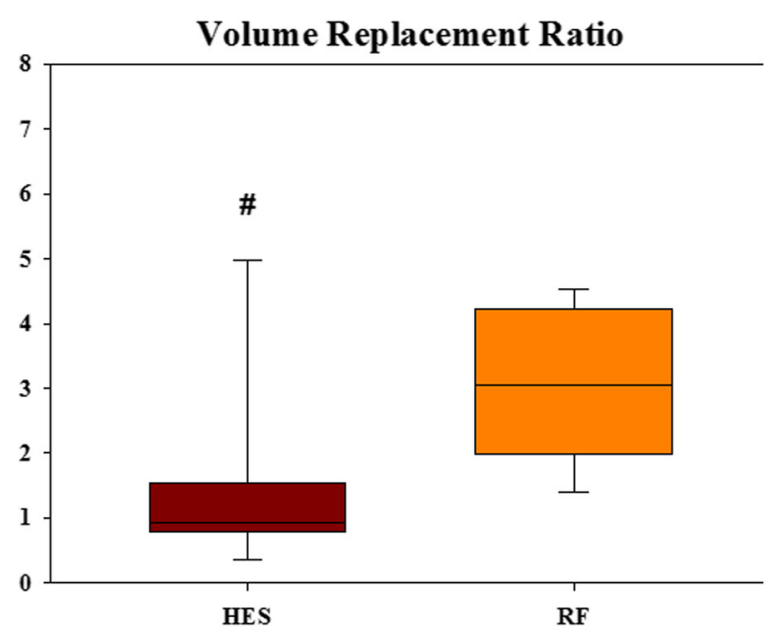

Fig. 3 Volume-replacement ratio: resuscitation fluid/total blood loss. Data are presented as median [IQR]. $p=0.002$
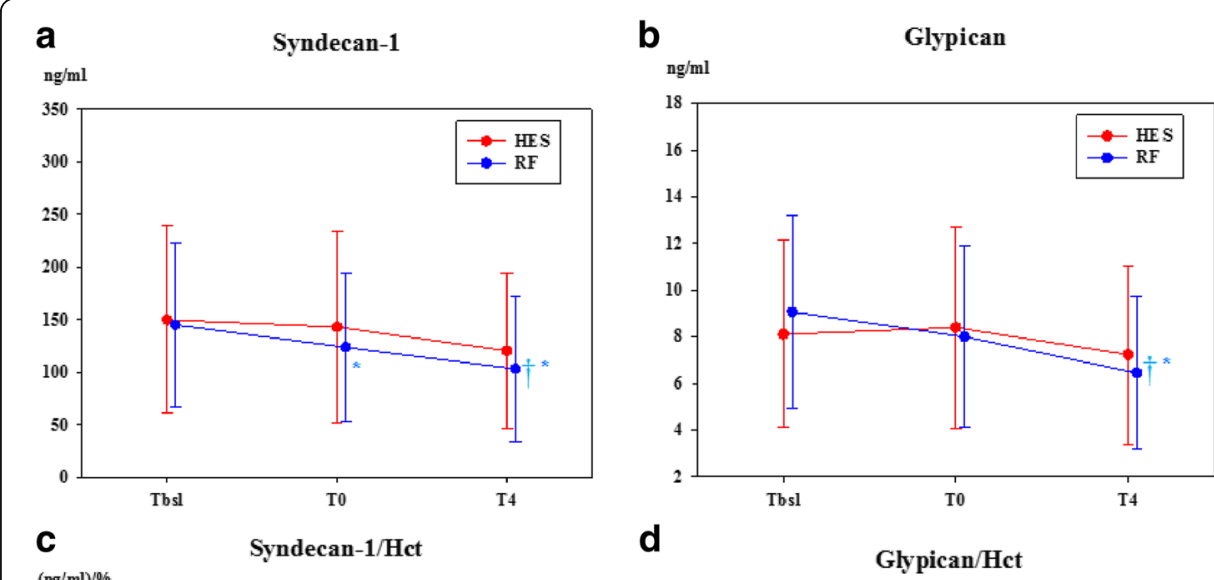

$(\mathrm{ng} / \mathrm{ml}) / \%$

$(\mathrm{ng} / \mathrm{ml}) \%$
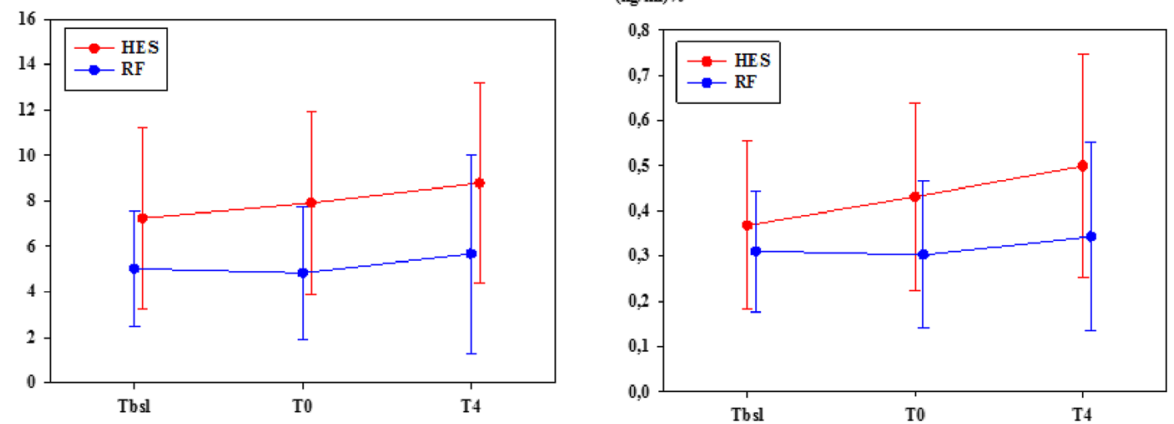

Fig. 4 Endothelial function. Plasma concentrations of syndecan-1 (a), glypican (b), syndecan-1 hematocrit ratio (c) and the glypican hematocrit ratio (d) are delineated. Data are presented as mean \pm standard deviation. HES $=$ colloid group, $\mathrm{RF}=$ crystalloid group. ${ }^{*} p<0.05$ significantly different from $T_{\mathrm{bs} \text {. }}{ }^{+} p<0.05$ significantly different from $T_{0}{ }^{*} p<0.05$ significantly different between groups 


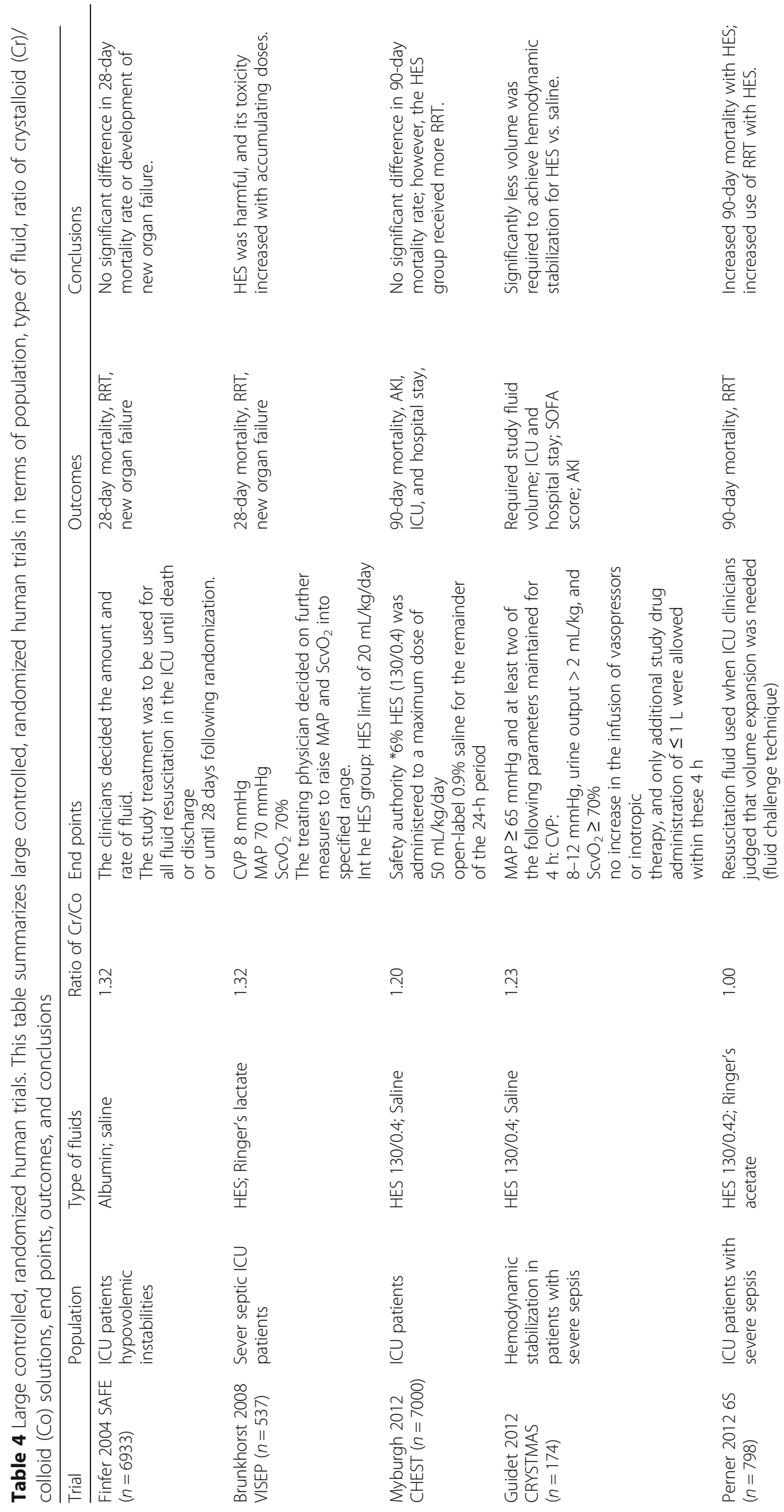




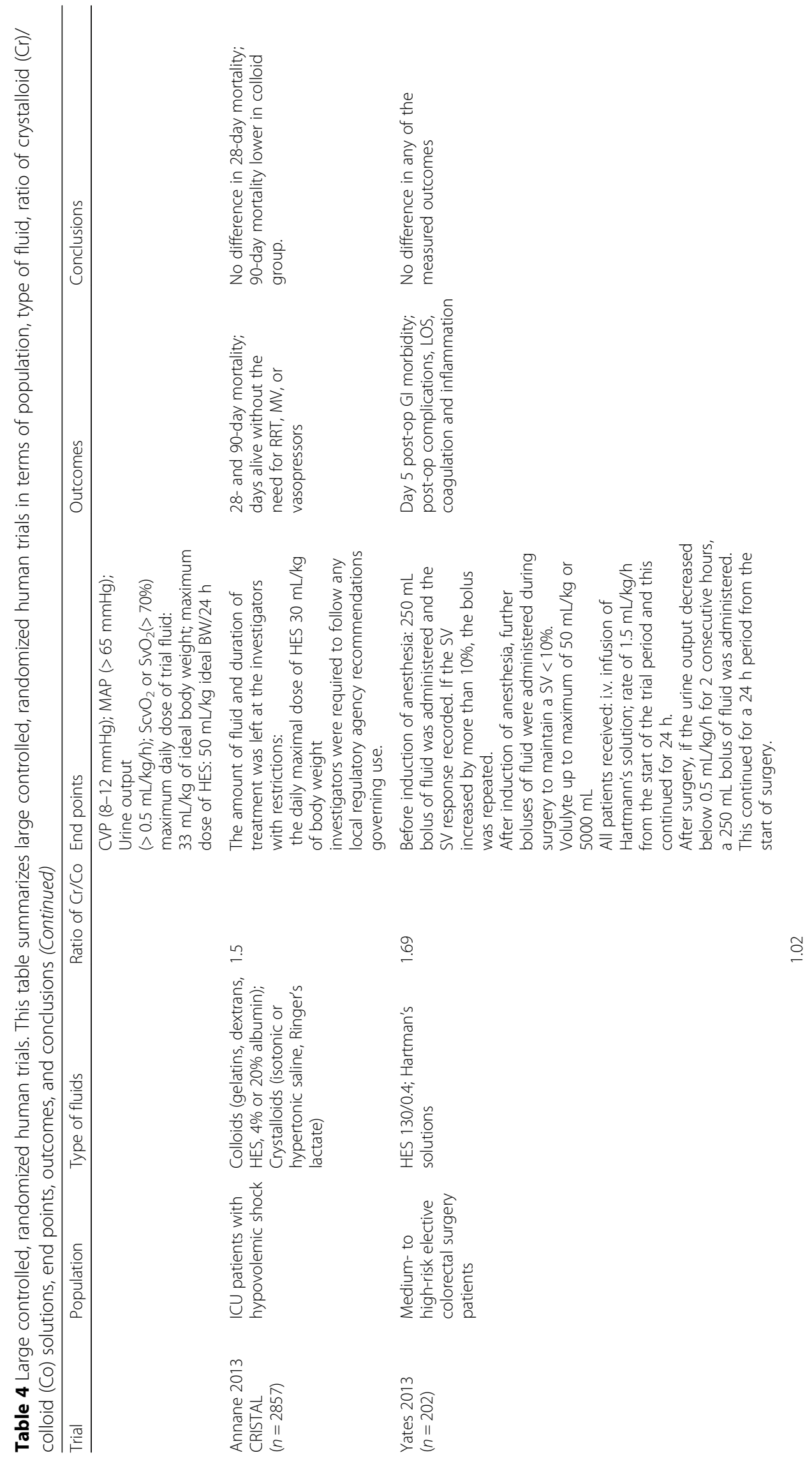




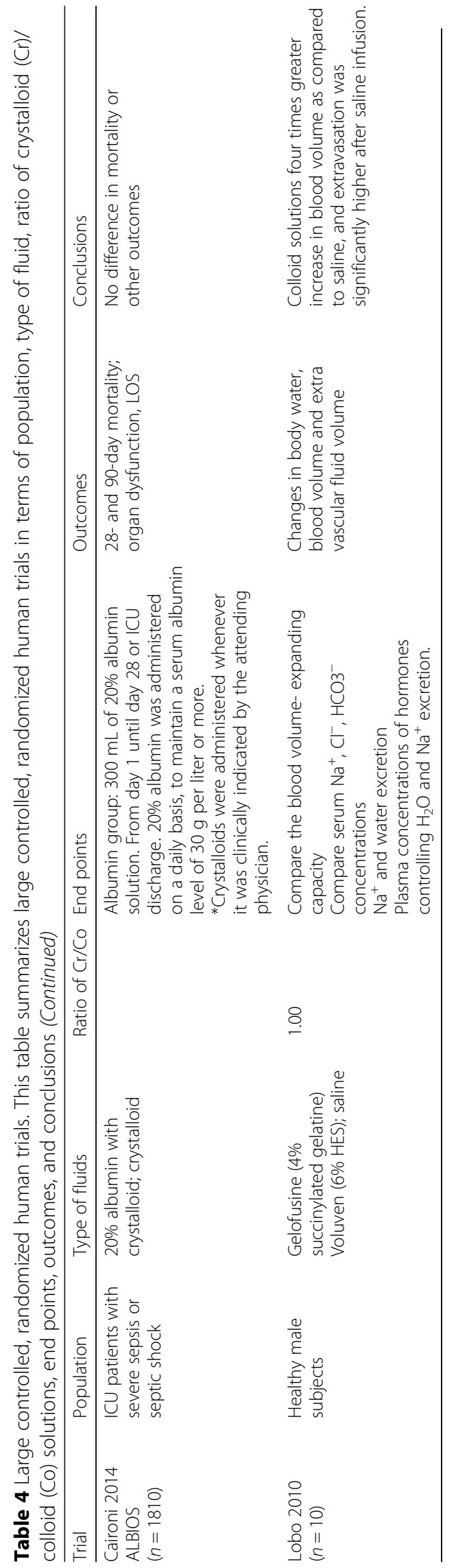


at all and hence received fluids unnecessarily. Nevertheless, the methods for which fluid administration was indicated in these trials, also reflects everyday practice, as was nicely shown by a large recent observational study [29]. In this survey by Cecconi et al., it was revealed that fluid therapy is mainly guided by inadequate indices. Therefore, one cannot exclude that in all the previously mentioned large trials, a considerable proportion of patients were not hypovolemic at all. This at least in part may explain the observed detrimental effects of hydroxyaethyl starches, as one cannot exclude that HES was administered to normovolemic patients; hence, its side/toxic effects were amplified. Furthermore, as it was shown in a human study with detailed blood and plasma volume assessment, different infusion volumes, infusion rates, plasma substitutes, or possibly different tracers for plasma volume measurement might lead to different results concerning the kinetics of fluid or colloid extravasation [30] .

Our intention was to perform a bleeding-resuscitation experiment with detailed hemodynamic monitoring, predefined end points, and a pragmatic protocol. During the experiment, hemodynamic changes did not show clinically relevant differences between the two groups. Kinetics of CI, SVI, MAP, HR, and GEVI showed similar patterns in both groups with significantly higher values in the HES group at the end of the experiment. The higher macro-hemodynamic values in the HES group may be due to the more rapid hemodynamic effects of colloids in general as compared to crystalloids. SVV and PPV almost doubled after bleeding in both groups and then returned to baseline values, with both being significantly lower in the HES group. Contractility, as indicated by dPmax values, also showed similar changes in both groups. In other words, we observed a similar hemodynamic course for these animals during the experiment, but the volume required was more than three times higher in the RF group. We detected elevated lactate and extravascular lung water (EVLW) levels from the start. This could have occurred during the preparation process, which may have caused some kind of distress. Nevertheless, EVLW did not reach extremely high values and regarding lactate, pigs can have higher blood lactate levels than humans, ranging from 0.5 to $5.5 \mathrm{mmol} / \mathrm{L}$ [31].

For decades, clinicians have based their choice of resuscitation fluids on Starling's well-known compartment model. According to his principle, capillaries and postcapillary venules act as a semipermeable membrane absorbing fluid from the interstitial space [32]; hence, the hydrostatic and oncotic pressure gradients across the semipermeable membrane are the principal determinants of transvascular exchange. However, this classic model has recently been challenged [33]. One of the most important reasons why the vasculature may behave differently than that described by Starling is the recently discovered role of the glycocalyx in the function of the endothelium.

A web of membrane-bound glycoproteins and proteoglycans on the luminal side of endothelium has been identified as the glycocalyx layer. This compartment consists of many highly sulfated GAG chains providing negative charge for the endothelium. Due to these electrostatic properties, the subglycocalyx space produces a colloid oncotic pressure that might be an important determinant of vascular permeability and thus fluid balance [34]. The structure and function of the endothelial glycocalyx varies substantially among different organ systems and is affected by inflammatory conditions, such as sepsis [35]. Theoretically, with an intact glycocalyx, the volume-replacement ratio is markedly different for crystalloids compared to colloids and may behave as 
suggested by Starling [36]. This is also supported by other studies, including our current experiment. In a recent trial on healthy volunteers, it was demonstrated that after $1000 \mathrm{ml}$ of crystalloid (isotonic saline) or colloid (gelatine and hydroxyaethyl starch) infusion, the latter caused a four times greater increase in blood volume compared to saline, and extravasation was significantly higher after saline infusion: saline $68 \%$, gelatine $21 \%$, and starch $16 \%$ [37]. In our experiment in healthy pigs, we also found similar differences between the volume expanding effects of RF and HES solutions. This suggests that during the early phase of bleeding, when theoretically the endothelium and the glycocalyx are intact, colloids have volume sparing effects compared to crystalloids.

\section{Limitations}

First of all, in this model, the course of bleeding took place relatively quickly, which was almost immediately followed by resuscitation. This scenario seldom takes place during daily routine. Therefore, the results of the current study can only be partially applied to clinical practice. Another limitation is that microcirculation and extravasation of fluid was not monitored or assessed in any way; hence, the measurement of certain glycocalyx degradation molecules can only be considered as indirect indicators of glycocalyx integrity. A more detailed evaluation would be necessary to prove our concept that Starling's theory worked in this model. Another limitation of our results is that animals remained alkalotic in the HES group as a result of unintentional hyperventilation at $T_{\mathrm{bs}}, T_{0}$, and $T_{1}$. However, whether it interfered with the results to any extent is difficult to tell. High EVLW and lactate levels, which were elevated and remained so throughout, indicate that animal preparation, which required a considerable length of time, was not as gentle as meant to and may have caused some distress. Finally, the long-term effects of fluid resuscitation on hemodynamics, renal function, and glycocalyx degradation were not assessed; therefore, our results cannot help the crystalloidcolloid debate as far as outcomes are concerned.

\section{Conclusions}

Our data provides experimental evidence that for the same hemodynamic effect, significantly more crystalloid than colloid solution is required in healthy pigs. Furthermore, the volume-replacement ratio was very similar to that described by Starling. Our data also suggests that the Starling's "three-compartment model" requires an intact endothelial glycocalyx. Therefore, the clinical importance of our results is that colloids may have a place in early resuscitation before the glycocalyx suffers impairment. Further studies are required, both experimental and clinical, in which, in addition to detailed hemodynamic monitoring, the function of the microcirculation and the glycolcalyx are also assessed.

\section{Acknowledgements}

We would like to thank the assistants, medical students, and staff at the Institute of Surgical Research and the Department of Anesthesiology and Intensive Therapy for their help. This work was supported by the National Research, Development and Innovation Office (NKFIH K116689). 
contributed to the data acquisition and critical revision of the manuscript. ZM contributed to the study conception and design and critical revision of the manuscript. All authors read and approved the final manuscript.

\section{Funding}

On behalf of all authors, the corresponding author states that the cost of animal experiments and glycocalyx degradation markers were covered by a grant of the National Research, Development and Innovation Office (NKFIH K116689).

\section{Ethics approval}

The experiments were performed on the EU Directive 2010/63/EU for the protection of animals used for experimental and other scientific purposes and carried out in strict adherence to the NIH guidelines for the use of experimental animals. The experimental project was approved by the National Scientific Ethical Committee on Animal Experimentation (National Competent Authority), Hungary, with license number: V./142/2013. The study was conducted in the research laboratory of the Institute of Surgical Research in a manner that did not inflict unnecessary pain or discomfort upon the animals.

\section{Competing interest}

The authors declare that they have no competing interests.

\section{Publisher's Note}

Springer Nature remains neutral with regard to jurisdictional claims in published maps and institutional affiliations.

\section{Author details}

${ }^{1}$ Faculty of Medicine; Institute of Surgical Research, University of Szeged, 6. Szőkefalvi-Nagy Béla st, Szeged 6720, Hungary. ${ }^{2}$ Faculty of Medicine; Department of Anaesthesiology and Intensive Therapy, University of Szeged, 6. Semmelweis st, Szeged 6725, Hungary.

Received: 31 July 2017 Accepted: 5 December 2017

Published online: 20 December 2017

\section{References}

1. Jabaley C, Dudaryk R (2014) Fluid resuscitation for trauma patients: crystalloids versus colloids. Curr Anesthesiol Rep 4:216-224. doi: 10.1007/s40140-014-0067-4

2. Qureshi SH, Rizvi SI, Patel NN, Murphy GJ (2015) Meta-analysis of colloids versus crystalloids in critically ill, trauma and surgical patients. Br J Surg:14-26. doi: 10.1002/bjs.9943

3. Bartels K, Thiele RH, Gan TJ (2013) Rational fluid management in today's ICU practice. Crit Care 17(Suppl 1):S6. doi: 10.1186/cc11504

4. Lira A, Pinsky MR (2014) Choices in fluid type and volume during resuscitation: impact on patient outcomes. Ann Intensive Care 4:38. doi: 10.1186/s13613-014-0038-4

5. Sauaia A, Moore FA, Moore EE et al (1995) Epidemiology of trauma deaths: a reassessment. J Trauma 38:185-193

6. Acosta JA, Yang JC, Winchell RJ et al (1998) Lethal injuries and time to death in a level I trauma center. J Am Coll Surg 186:528-533

7. Hoyt DB, Bulger EM, Knudson MM et al (1994) Death in the operating room: an analysis of a multi-center experience. J Trauma 37:426-432

8. Woodcock TE, Woodcock TM (2012) Revised Starling equation and the glycocalyx model of transvascular fluid exchange: an improved paradigm for prescribing intravenous fluid therapy. Br J Anaesth 108:384-394. doi: 10. 1093/bja/aer515

9. Chappell D, Jacob M, Hofmann-kiefer K et al (2017) A rational approach to perioperative fluid management. Anaesthesiology 109:723-740

10. Annane D (2013) Effects of fluid resuscitation with colloids vs crystalloids on mortality in critically ill patients presenting with hypovolemic shock. JAMA:1-9. doi: 10.1001/jama.2013.280502

11. Brunkhorst FM, Engel C, Bloos F et al (2008) Intensive insulin therapy and pentastarch resuscitation in severe sepsis. N Engl J Med 358:125-139. doi: 10.1056/NEJMoa070716

12. Perner A, Haase N, Guttormsen AB et al (2012) Hydroxyethyl starch 130/0.42 versus Ringer's acetate in severe sepsis. N Engl J Med 367:124-134. doi: 10.1056/NEJMoa1204242

13. Myburgh JA, Finfer S, Bellomo R et al (2012) Hydroxyethyl starch or saline for fluid resuscitation in intensive care. N Engl J Med 367:1901-1911. doi: 10.1056/NEJMoa1209759

14. Guidet B, Martinet O, Boulain T et al (2012) Assessment of hemodynamic efficacy and safety fluid replacement in patients with severe sepsis : the CRYSTMAS study. Crit Care 16:R94. doi: 10.1186/11358

15. Finfer S, Bellomo R, Boyce $\mathrm{N}$ et al (2004) A comparison of albumin and saline for fluid resuscitation in the intensive care unit. N Engl J Med 350:2247-2256. doi: 10.1186/cc3006

16. Nelson A, Berkestedt I, Schmidtchen A et al (2008) Increased levels of glycosaminoglycans during septic shock: relation to mortality and the antibacterial actions of plasma. Shock 30:623-627. doi: 10.1097/SHK. 0b013e3181777da3

17. Chappell D, Westphal M, Jacob M (2009) The impact of the glycocalyx on microcirculatory oxygen distribution in critical illness. Curr Opin Anaesthesiol 22:155-162. doi: 10.1097/ACO.0b013e328328d1b6

18. Johansson PI, Sørensen AM, Perner AWK et al (2011) Elderly trauma patients have high circulating noradrenaline levels but attenuated release of adrenaline, platelets, and leukocytes in response to increasing injury severity. Ann Surg 254:194. doi: 10.1097/SLA.0b013e318226113d 
19. Johansson PI, Stensballe J, Rasmussen LS, Ostrowski SR (2012) High circulating adrenaline levels at admission predict increased mortality after trauma. J Trauma Acute Care Surg 72:428-436

20. Marechal X, Favory R, Joulin $O$ et al (2008) Endothelial glycocalyx damage during endotoxemia coincides with microcirculatory dysfunction and vascular oxidative stress. Shock 29:572-576. doi: 10.1097/SHK.0b013e318157e926

21. Nieuwdorp M, Mooij HL, Kroon J et al (2006) Endothelial glycocalyx damage coincides with microalbuminuria in type 1 diabetes. Diabetes 55:1127-1132

22. Tánczos K, Németh M, Trásy D et al (2015) Goal-directed resuscitation aiming cardiac index masks residual hypovolemia: an animal experiment. Biomed Res Int 2015:1-8. doi: 10.1155/2015/160979

23. Németh M, Tánczos K, Demeter $G$ et al (2014) Central venous oxygen saturation and carbon dioxide gap as resuscitation targets in a hemorrhagic shock. Acta Anaesthesiol Scand 58:611-619. doi: 10.1111/aas.12312

24. EMANUEL R, BRYANT N, SUZANNE H, et al (2001) The New England Journal of Medicine Early goal-directed therapy in the treatment of severe sepsis and septic shock. N Engl J Med 345:1368-1377

25. Dellinger RP, Levy MM, Rhodes A et al (2013) Surviving sepsis campaign. Crit Care Med 41:580-637. doi: 10.1097/ CCM.0b013e31827e83af

26. Soni N (2009) British Consensus Guidelines on Intravenous Fluid Therapy for Adult Surgical Patients (GIFTASUP): Cassandra's view. Anaesthesia 64:235-238. doi: 10.1111/j.1365-2044.2009.05886_1.x

27. Advanced Trauma Life Support. https://www.facs.org/quality-programs/trauma/atls. Accessed 29 May 2017

28. Linton RAF, Linton NWF, Kelly F (2002) Is clinical assessment of the circulation reliable in postoperative cardiac. J Cardiothorac Vasc Anesth 16:4-7. doi: 10.1053/jcan.2002.29631

29. Cecconi M, Hofer C, Teboul JL et al (2015) Fluid challenges in intensive care: the FENICE study: a global inception cohort study. Intensive Care Med 41:1529-1537. doi: 10.1007/s00134-015-3850-x

30. Rehm M, Haller M, Orth V, Kreimeier U, Jacob M, Dressel H, Mayer S, Brechtelsbauer H FU (2017) Changes in blood volume and hematocrit during acute preoperative volume loading with $5 \%$ albumin or $6 \%$ hetastarch solutions in patients before radical hysterectomy. Anaesthesia L:849-856

31. Hofmaier F, Dinger K, Braun R (2013) Short report range of blood lactate values in farm pigs prior to experimental surgery. Lab Anim 47:130-132

32. Starling EH (1896) On the absorption of fluids from the connective tissue spaces. J Physiol 19:312-326

33. Levick JR, Michel CC (2010) Microvascular fluid exchange and the revised Starling principle. Cardiovasc Res 87: 198-210. doi: 10.1093/cvr/cvq062

34. Ince C (2014) The rationale for microcirculatory guided fluid therapy. Curr Opin Crit Care 20:301-308. doi: 10.1097/ MCC 0000000000000091

35. Lee WL, Slutsky AS (2010) Sepsis and endothelial permeability. N Engl J Med 363:689-691. doi: 10.1056/ NEJMcibr1007320

36. Kolářová H, Ambrůzová B, Svihálková Šindlerová L et al (2014) Modulation of endothelial glycocalyx structure under inflammatory conditions. Mediat Inflamm 2014:694312. doi: 10.1155/2014/694312

37. Honore PM, Jamez J, Wauthier M et al (2000) Prospective evaluation of short-term, high-volume isovolemic hemofiltration on the hemodynamic course and outcome in patients with intractable circulatory failure resulting from septic shock. Crit Care Med 28:3581-3587

Submit your manuscript to a SpringerOpen ${ }^{\circ}$ journal and benefit from:

- Convenient online submission

- Rigorous peer review

- Open access: articles freely available online

High visibility within the field

Retaining the copyright to your article

Submit your next manuscript at $>$ springeropen.com 